Pacific

Journal of

Mathematics

ABSOLUTE CONTINUITY BETWEEN THE WIENER AND STATIONARY GAUSSIAN MEASURES

U. KEICH 


\title{
ABSOLUTE CONTINUITY BETWEEN THE WIENER AND STATIONARY GAUSSIAN MEASURES
}

\author{
U. KEICH
}

It is known that the entropy distance between two Gaussian measures is finite if, and only if, they are absolutely continuous with respect to one another. Shepp (1966) characterized the correlations corresponding to stationary Gaussian measures that are absolutely continuous with respect to the Wiener measure. By analyzing the entropy distance, we show that one of his conditions, involving the spectrum of an associated operator, is essentially extraneous, providing a simple criterion for finite entropy distance in this case.

\section{Introduction.}

Let $C[1-\tau, 1+\tau]$ (where $0<\tau<1$ ) denote the space of continuous functions on $[1-\tau, 1+\tau]$. A standard Brownian motion observed between times $1-\tau$ and $1+\tau$ induces on $C[1-\tau, 1+\tau]$ the Wiener measure $W^{\tau}$. As a Gaussian measure, it is characterized by its correlation $R(t, s)=t \wedge s$ for $t, s \in[1-\tau, 1+\tau]$, and by its vanishing mean.

A Gaussian measure, $Q^{\tau}$, on $C[1-\tau, 1+\tau]$ is stationary if its mean is constant and its correlation is a Töeplitz function. That is, with $X \in$ $C[1-\tau, 1+\tau]$ being the sample path,

$$
\mu_{t} \stackrel{d}{=} E^{Q^{\tau}} X_{t} \equiv \text { const. } \quad t \in[1-\tau, 1+\tau],
$$

and

$$
S(t, s) \stackrel{d}{=} E^{Q^{\tau}} X_{t} X_{s}-\mu^{2}=S(t-s) \quad t, s \in[1-\tau, 1+\tau],
$$

where $S$ denotes both the correlation $S(t, s)$, and the auto-correlation $S(r)$ with $r=t-s \in[-2 \tau, 2 \tau]$. Krein [4] showed that $S(r)$ can always be extended to $\mathbb{R}$ as a continuous positive-definite function, thereby providing an extension of $Q^{\tau}$ to a stationary Gaussian measure, $Q$, on $C(\mathbb{R})$.

We would like to characterize the stationary measures $Q^{\tau}$ which are absolutely continuous with respect to $W^{\tau}$. Since the measures are Gaussian, this question can be settled in terms of the mean $\mu$ and the correlation $S$ of $Q^{\tau}$. Furthermore, for $\tau<1$, the Brownian paths $b_{t}$ and $b_{t}+\mu$ with $t \in[1-\tau, 1+\tau]$ induce measures that are absolutely continuous with respect 
to one another, and therefore the constant mean of $Q$ is irrelevant to the question of absolute continuity.

Shepp provides the following necessary and sufficient conditions for a stationary Gaussian measure $Q^{\tau_{0}}$ with correlation $S$ to be absolutely continuous with respect to $W^{\tau_{0}}[\mathbf{5}]$ :

(i) On $\left(0,2 \tau_{0}\right), S^{\prime}$ is absolutely continuous and $S^{\prime \prime}$ satisfies

$$
\int_{0}^{2 \tau_{0}} S^{\prime \prime}(t)^{2}\left(2 \tau_{0}-t\right) d t<\infty .
$$

(ii) $S^{+}(0)=-1 / 2 \quad$ (the derivative from the right).

(iii) $-1 \notin \sigma(F)$, where $\sigma(F)$ is the spectrum of the integral operator defined by the kernel

$$
F \stackrel{d}{=} \frac{\partial}{\partial s} \frac{\partial}{\partial t}\left[S(t-s)-\frac{S(t) S(s)}{S(0)}-t \wedge s\right] \quad t, s \in\left[0,2 \tau_{0}\right] .
$$

Shepp gives an example showing that (iii) is essential:

$$
S(r) \stackrel{d}{=} \frac{1}{4}-\frac{|r|}{2} \quad \text { for } \quad-2 \tau_{0} \leq r \leq 2 \tau_{0} .
$$

Here (i) and (ii) hold for any $\tau_{0}>0$, but (iii) is valid only if $\tau_{0}<\frac{1}{2}$ $\left(\tau_{0} \leq \frac{1}{2}\right.$ is required for $S$ to be positive-definite). Indeed, with $\tau_{0}=\frac{1}{2}$ and $X \in C\left[1-\tau_{0}, 1+\tau_{0}\right]$, we have $X_{1 / 2}=-X_{3 / 2}$ a.s. $d Q$, thus ruling out absolute continuity with respect to $W^{\tau_{0}}$. However, a closer look at this example yields two interesting facts for $\tau_{0}=\frac{1}{2}$ :

- There exists only one positive-definite extension of $S$ from $\left[-2 \tau_{0}, 2 \tau_{0}\right]$ to $\mathbb{R}([4])$.

- $S^{\prime \prime}$ does not exist at $r=2 \tau_{0}$.

Both observations turn out to be the rule whenever (iii) is violated. This allows us to rid ourselves of the third condition in Shepp's theorem by paying a small price: (i) and (ii) suffice for absolute continuity for $\tau<\tau_{0}$. The precise statement is:

Theorem 1. If $Q^{\tau_{0}}$, a stationary Gaussian measure with correlation $S$, is absolutely continuous with respect to $W^{\tau_{0}}\left(0<\tau_{0}<1\right)$, then (i) and (ii) hold. If, on the other hand, (i) and (ii) hold, then for any $0<\tau<\tau_{0}, Q^{\tau}$ is absolutely continuous with respect to $W^{\tau}$.

\section{Remarks.}

- A simple scaling argument shows that the time interval can be centered about any point (not necessarily 1).

- Condition (ii) guarantees that the measure $Q$ is supported on paths with the same quadratic variation as that of Brownian motion. This must be so, as it is an "almost sure" property of the Brownian path. 
We next provide a brief overview of the proof which is entirely different from Shepp's; it relies on our ability to estimate the entropy distance between $Q^{\tau}$ and $W^{\tau}$. Let $P$ and $Q$ be Gaussian measures on $C[1-\tau, 1+\tau]$. It is known that the entropy distance between $P$ and $Q, H(P, Q)$, is finite if and only if the two measures are absolutely continuous with respect to one another; in that case:

$$
H(P, Q)=E^{P}\left(\log \frac{d P}{d Q}\right)+E^{Q}\left(\log \frac{d Q}{d P}\right),
$$

where $E^{P}$ is the expectation under the measure $P$ and $\frac{d P}{d Q}$ is the RadonNikodym derivative of $P$ with respect to $Q$ (see e.g. [3]).

Let $P_{n}$ and $Q_{n}$ be the restrictions of $P$ and $Q$ to $n+1$ equally spaced points in $[1-\tau, 1+\tau]$, with correlations $R_{n}$ and $S_{n}$. Then

$$
H(P, Q)=\varlimsup{ }\left(P_{n}, Q_{n}\right) .
$$

Let $K_{n}$ be a root of $R_{n}$, i.e., $R_{n}=K_{n} K_{n}^{*}$, and let $T_{n}=K_{n}^{-1} S_{n} K_{n}^{-*}$, with $K^{-*}$ being a short for $\left(K^{-1}\right)^{*}$. If $\lambda_{i}^{n}$ are the eigenvalues of $T_{n}$, then

$$
H\left(P_{n}, Q_{n}\right)=\frac{1}{2} \sum_{i=0}^{n} \frac{\left(\lambda_{i}^{n}-1\right)^{2}}{\lambda_{i}^{n}} .
$$

Thus, $H\left(W^{\tau_{0}}, Q^{\tau_{0}}\right)<\infty$ implies

$$
\sup _{\tau \leq \tau_{0}} \varlimsup \sum\left(\lambda_{i}^{n}-1\right)^{2}<\infty .
$$

In the case of $W^{\tau_{0}}(R=t \wedge s), R_{n}^{-1}$ is essentially a second order difference operator so we can choose $K_{n}$ so that $K_{n}^{-1}$ is basically a first order difference operator. Thus, the typical entry in $T_{n}=K_{n}^{-1} S_{n} K_{n}^{-*}$ is a second order difference of the sampled Töeplitz correlation $S_{n}$. Add to this the identity

$$
\varlimsup \sum\left(\lambda_{i}^{n}-1\right)^{2}=\varlimsup \lim \operatorname{Tr}\left(T_{n}-I\right)^{2},
$$

and you will understand how (1) implies the existence of $S^{\prime}$ and $S^{\prime \prime}$ on $\left(0,2 \tau_{0}\right)$ as in (i) and (ii) of Theorem 1.

This is the easier half of that theorem. As for the other half, with our choice of $K_{n}$ we can readily show that (1) follows from (i) and (ii) of Theorem 1. It is left to prove that for $\tau<\tau_{0}, \inf _{i, n} \lambda_{i}^{n}(\tau)>0$ (this is the analogue of Shepp's third condition, which we omit). Indeed, if $\inf _{i, n} \lambda_{i}^{n}(\tau)=0$, then there exist $\lambda_{k} \longrightarrow 0$ and $\boldsymbol{v}_{k} \in \mathbb{R}^{n_{k}}$, such that

$$
T_{n_{k}} \boldsymbol{v}_{k}=\lambda_{k} \boldsymbol{v}_{k} \text {. }
$$

Appropriately embedded in $\mathcal{H} \stackrel{d}{=} \mathbb{R} \oplus L^{2}[0,2 \tau], \boldsymbol{v}_{k}$ converge strongly to a non-zero limit $(\beta, f) \in \mathcal{H}$ with the property that almost surely $d Q$ :

$$
\beta X_{0}+\int_{0}^{2 \tau} f(t) d X_{t}=0
$$


where $Q$ is a stationary extension of $Q^{\tau}$ to $C(\mathbb{R})$ and $X_{t}$ is the sample path. As we show, this implies that $S$ has a unique positive-definite extension, from $[-2 \tau, 2 \tau]$ to $\mathbb{R}$. This unique extension has the property that $S^{\prime \prime} \notin$ $L^{2}(0,2 \tau+\varepsilon)$ for any $\varepsilon>0$, contradicting (i).

\section{Proof of Theorem 1.}

The space $C_{0} \stackrel{d}{=} C\left[1-\tau_{0}, 1+\tau_{0}\right]$, is equipped with the $\sigma$-field $\mathcal{F}$ generated by the cylinder sets. Wiener measure (denoted by $W$ ) is defined on that space, so any measure $Q$ that is absolutely continuous with respect to it must live there. On the other hand, an application of Kolmogorov-Čensov shows that a correlation $S$ subject to (i) and (ii) of theorem 1 defines a probability measure $Q$ on $C_{0}$.

Remark. Since the mean of $Q$ is irrelevant to our problem it may be assumed to vanish.

Let $P$ and $Q$ be Gaussian measures on $C_{0}$. For $0<\tau \leq \tau_{0}$, let $P^{\tau}, Q^{\tau}$ and $\mathcal{F}^{\tau}$ be the restrictions of $P, Q$, respectively $\mathcal{F}$, to $C[1-\tau, 1+\tau]$. Let $\mathcal{F}_{n}^{\tau}$ be the $\sigma$-field obtained by sampling the paths on $[1-\tau, 1+\tau]$ at $n+1$ equally spaced points. Let $P_{n}^{\tau}$ and $Q_{n}^{\tau}$ be the restrictions of $P^{\tau}$ and $Q^{\tau}$ to that $\sigma$-field. Let

$$
H_{n}^{\tau} \stackrel{d}{=} H\left(P_{n}^{\tau}, Q_{n}^{\tau}\right) .
$$

Since $\mathcal{F}^{\tau}$ is generated by $\bigcup \mathcal{F}_{n}^{\tau}$, it follows that $H\left(P^{\tau}, Q^{\tau}\right)=\varlimsup \lim H_{n}^{\tau}[\mathbf{3}]$.

A first step is to express the entropy $H_{n}^{\tau}$ in terms of the $(n+1) \times(n+1)$ sampled correlations, $S_{n}^{\tau}$ and $R_{n}^{\tau}$.

Claim 2.1. Let $R$ and $S$ be the $m \times m$ correlation matrices of the 0 -mean Gaussian measures $P$, respectively $Q$ on $\mathbb{R}^{m}$, and let $K$ be a root of $R$ so that $R=K K^{*}$. Then

$$
H(P, Q)=\frac{1}{2} \operatorname{Tr}\left(K^{-1} S K^{-*}+K^{*} S^{-1} K-2 I\right) .
$$

Proof.

$$
\begin{aligned}
E^{Q} \log \frac{d Q}{d P} & =E^{Q} \log \left\{\frac{\sqrt{\operatorname{det} R}}{\sqrt{\operatorname{det} S}} \exp \left[-\frac{1}{2}\left\langle\left(S^{-1}-R^{-1}\right) \boldsymbol{x}, \boldsymbol{x}\right\rangle\right]\right\} \\
& =-\frac{1}{2} \log \operatorname{det} R^{-1} S-\frac{1}{2} E^{Q}\left\langle\left(S^{-1}-R^{-1}\right) \boldsymbol{x}, \boldsymbol{x}\right\rangle,
\end{aligned}
$$

with $\boldsymbol{x} \in \mathbb{R}^{m}$.

If $A$ is any $m \times m$ matrix, then

$$
E^{Q}\langle A \boldsymbol{x}, \boldsymbol{x}\rangle=E^{Q} \sum_{i, j} a_{i j} \boldsymbol{x}_{i} \boldsymbol{x}_{j}=\sum_{i} \sum_{j} a_{i j} s_{j i}=\operatorname{Tr} A S,
$$


SO

$$
E^{Q} \log \frac{d Q}{d P}=-\frac{1}{2} \log \operatorname{det} R^{-1} S+\frac{1}{2} \operatorname{Tr}\left(R^{-1} S-I\right) .
$$

The same applies to $E^{P} \log \frac{d P}{d Q}$ so the entropy is:

$$
H=\frac{1}{2} \operatorname{Tr}\left(R^{-1} S+S^{-1} R-2 I\right)
$$

The claim follows from $\operatorname{Tr} A B=\operatorname{Tr} B A$.

Let $K$ be a root of $R_{n}^{\tau}$ as above and put

$$
T \stackrel{d}{=} K^{-1} S K^{-*} \text {. }
$$

Then $T$ is symmetric, positive definite, and $T^{-1}=K^{*} S^{-1} K$, so from claim 2.1 ,

$$
H_{n}^{\tau}=\frac{1}{2} \operatorname{Tr}\left(T+T^{-1}-2 I\right) .
$$

In terms of the (positive) eigenvalues $\lambda_{i}^{n}=\lambda_{i}^{n}(\tau)$, of $T$ :

$$
H_{n}^{\tau}=\frac{1}{2} \sum_{i=0}^{n} \frac{\left(\lambda_{i}^{n}-1\right)^{2}}{\lambda_{i}^{n}}
$$

For $0<\tau \leq \tau_{0}, H^{\tau} \leq H \stackrel{d}{=} H^{\tau_{0}}$, and since $H^{\tau}=\varlimsup \lim H_{n}^{\tau}$, it follows that

$$
H<\infty \quad \text { only if } \quad \varlimsup_{n} \sum_{i}\left(\lambda_{i}^{n}-1\right)^{2} \leq C<\infty,
$$

where $C$ is a constant which depends on $\tau_{0}$. Note that

$$
\sum_{i=0}^{n}\left(\lambda_{i}^{n}-1\right)^{2}=\operatorname{Tr}(T-I)^{2}=\sum_{i \neq j} t_{i j}^{2}+\sum_{i}\left(t_{i i}-1\right)^{2} .
$$

Let $\delta=\delta_{n}^{\tau}=2 \tau / n$ be the mesh of the partition. Returning to the case of $R=t \wedge s$ and a stationary correlation $S=S(t-s)$, we choose

$$
K \stackrel{d}{=}\left(\begin{array}{cccccc}
\sqrt{1-\tau} & 0 & 0 & 0 & \ldots & 0 \\
\sqrt{1-\tau} & \sqrt{\delta} & 0 & 0 & \ldots & 0 \\
\sqrt{1-\tau} & \sqrt{\delta} & \sqrt{\delta} & 0 & \ldots & 0 \\
\vdots & \vdots & & \ddots & & \\
\sqrt{1-\tau} & \sqrt{\delta} & \sqrt{\delta} & \ldots & \sqrt{\delta} & 0 \\
\sqrt{1-\tau} & \sqrt{\delta} & \sqrt{\delta} & \ldots & \sqrt{\delta} & \sqrt{\delta}
\end{array}\right) .
$$


It is easy to verify that $R_{n}^{\tau}=K K^{*}$ and that

$$
K^{-1}=\left(\begin{array}{cccccc}
\frac{1}{\sqrt{1-\tau}} & 0 & 0 & 0 & \ldots & 0 \\
-\frac{1}{\sqrt{\delta}} & \frac{1}{\sqrt{\delta}} & 0 & 0 & \ldots & 0 \\
0 & -\frac{1}{\sqrt{\delta}} & \frac{1}{\sqrt{\delta}} & 0 & \ldots & 0 \\
\vdots & & \ddots & & & \\
0 & 0 & \ldots & -\frac{1}{\sqrt{\delta}} & \frac{1}{\sqrt{\delta}} & 0 \\
0 & 0 & \ldots & & -\frac{1}{\sqrt{\delta}} & \frac{1}{\sqrt{\delta}}
\end{array}\right) .
$$

It follows that, with $S_{k}=S(k \delta)$,

(5)

$$
T=\left(\begin{array}{cccccc}
\frac{S_{0}}{1-\tau} & \frac{1}{\sqrt{1-\tau}} \frac{S_{1}-S_{0}}{\sqrt{\delta}} & \frac{1}{\sqrt{1-\tau}} \frac{S_{2}-S_{1}}{\sqrt{\delta}} & \frac{1}{\sqrt{1-\tau}} \frac{S_{3}-S_{2}}{\sqrt{\delta}} & \ldots & \frac{1}{\sqrt{1-\tau}} \frac{S_{n}-S_{n-1}}{\sqrt{\delta}} \\
* & 2 \frac{S_{0}-S_{1}}{\delta} & \frac{2 S_{1}-S_{0}-S_{2}}{\delta} & \frac{2 S_{2}-S_{1}-S_{3}}{\delta} & \ldots & \frac{2 S_{n-1}-S_{n-2}-S_{n}}{\delta} \\
* & * & 2 \frac{S_{0}-S_{1}}{\delta} & \frac{2 S_{1}-S_{0}-S_{2}}{\delta} & \ldots & \frac{2 S_{n-2}-S_{n-3}-S_{n-1}}{\delta} \\
* & * & * & 2 \frac{S_{0}-S_{1}}{\delta} & \ddots & \vdots \\
* & * & * & & \ddots & \frac{2 S_{1}-S_{0}-S_{2}}{\delta} \\
* & * & & & & 2 \frac{S_{0}-S_{1}}{\delta}
\end{array}\right)
$$

where the $*$ 's are filled in according to the symmetry of $T$ (note the Töeplitz $n \times n$ sub-matrix). Using (4) we get:

$$
\begin{aligned}
\sum_{i=0}^{n}\left(\lambda_{i}^{n}-1\right)^{2}=\left(\frac{S_{0}}{1-\tau}-1\right)^{2} & +n\left(2 \frac{S_{0}-S_{1}}{\delta}-1\right)^{2} \\
& +2 \frac{1}{1-\tau} \sum_{k=1}^{n} \frac{\left(S_{k}-S_{k-1}\right)^{2}}{\delta} \\
& +2 \sum_{k=1}^{n-1}\left(\frac{2 S_{k}-S_{k-1}-S_{k+1}}{\delta}\right)^{2}(n-k) .
\end{aligned}
$$

Assume now that $Q^{\tau_{0}}$ is absolutely continuous with respect to $W^{\tau_{0}}$. Using (3) and the last equation, with $\delta=2 \tau_{0} / n$, we find:

$$
\begin{gathered}
M_{0}=\sup _{\tau \leq \tau_{0}} \sup _{n} n\left(2 \frac{S_{0}-S_{1}}{2 \tau / n}-1\right)^{2}<\infty, \\
M_{1}=\sup _{n} \sum_{k=1}^{n}\left(S_{k}-S_{k-1}\right)^{2} \frac{1}{\delta}<\infty,
\end{gathered}
$$


and

$$
M_{2}=\sup _{n} \sum_{k=1}^{n-1}\left(\frac{2 S_{k}-S_{k-1}-S_{k+1}}{\delta}\right)^{2}(n-k)<\infty .
$$

It follows from (7) that $S$ is continuous from the right at 0 , and since it is symmetric and positive definite, $S$ is also uniformly continuous on its domain (see e.g. [1], p. 191).

Claim 2.2. $S$ is an absolutely continuous function.

Proof. Let

$$
S_{n}(r) \stackrel{d}{=} \sum_{k} S(k \delta) 1_{[k \delta,(k+1) \delta)}(r)
$$

and

$$
f_{n}(r) \stackrel{d}{=} \frac{S_{n}(r+\delta)-S_{n}(r)}{\delta} .
$$

It follows from (8) that, with $\delta=2 \tau_{0} / n$ as before, $\int_{0}^{2 \tau_{0}} f_{n}{ }^{2} \leq M_{1}$, so there exists a subsequence $f_{n_{k}}$ converging weakly in $L^{2}$ to some function $f$. If for any smooth compactly supported $\varphi$ on $\left(0,2 \tau_{0}\right)$,

$$
\int_{0}^{2 \tau_{0}} f \varphi=-\int_{0}^{2 \tau_{0}} S \varphi^{\prime}
$$

then standard Sobolev type arguments show that $S$ is absolutely continuous (and $S^{\prime}=f$ ). To prove (10), note that for sufficiently large $n$ ( $\varphi$ being compactly supported),

$$
\begin{aligned}
\int_{0}^{2 \tau_{0}} f_{n_{k}}(t) \varphi(t) d t & =\int_{0}^{2 \tau_{0}} \frac{S_{n_{k}}(t+\delta)-S_{n_{k}}(t)}{\delta} \varphi(t) d t \\
& =-\int_{0}^{2 \epsilon_{0}} S_{n_{k}}(t) \frac{\varphi(t)-\varphi(t-\delta)}{\delta} d t .
\end{aligned}
$$

Since $S$ is continuous and $\varphi$ is smooth, by letting $k \rightarrow \infty$ in the last equation we get (10).

It is a corollary of the last claim and (7) that $S^{+}(0)=-\frac{1}{2}$, as in (ii) of the theorem.

Claim 2.3. $S^{\prime}$ is absolutely continuous on $\left(0,2 \tau_{0}\right)$ and $\int_{0}^{2 \tau_{0}} S^{\prime \prime}(t)^{2}\left(2 \tau_{0}-\right.$ t) $d t<\infty$.

Proof. With a slight abuse of notation, define

$$
S_{n}^{\prime}(r) \stackrel{d}{=} \sum_{k}\left(\frac{1}{\delta} \int_{k \delta}^{(k+1) \delta} S^{\prime}(\eta) d \eta\right) 1_{[k \delta,(k+1) \delta)}(r),
$$


and let

$$
\begin{aligned}
g_{n}(r) & \stackrel{d}{=} \frac{S_{n}^{\prime}(r+\delta / 2)-S_{n}^{\prime}(r-\delta / 2)}{\delta} \\
& =\sum_{k} \frac{S((k+1) \delta)+S((k-1) \delta)-2 S(k \delta)}{\delta^{2}} 1_{[k \delta,(k+1) \delta)}(r) .
\end{aligned}
$$

Since, by (9),

$$
\begin{aligned}
& \int_{0}^{2 \tau_{0}} g_{n}(t)^{2}\left(2 \tau_{0}-t\right) d t \\
& =\sum\left[\frac{S((k+1) \delta)+S((k-1) \delta)-2 S(k \delta)}{\delta^{2}}\right]^{2}\left(2 \tau_{0}-k \delta\right) \leq M_{2},
\end{aligned}
$$

there exists a subsequence $\left\{g_{n_{k}}\right\}$ that converges weakly to a limit $g$ in $L^{2}\left(\left(2 \tau_{0}-t\right) d t\right)$. Hence, for every proper subinterval $I \subset\left(0,2 \tau_{0}\right),\left\{g_{n_{k}}\right\}$ converges weakly in $L^{2}(I, d t)$. Again,

$$
\int_{0}^{2 \tau_{0}} g \varphi=-\int_{0}^{2 \tau_{0}} S^{\prime} \varphi^{\prime}
$$

will show $S^{\prime}$ is absolutely continuous with $g=S^{\prime \prime}$. For sufficiently large $n$,

$$
\int_{0}^{2 \tau_{0}} g_{n_{k}} \varphi=-\int_{0}^{2 \tau_{0}} S_{n_{k}}^{\prime}(t) \frac{\varphi(t+\delta / 2)-\varphi(t-\delta / 2)}{\delta} d t .
$$

Since $S_{n_{k}}^{\prime} \longrightarrow S^{\prime}$ in $L^{1}$ and $\varphi$ is smooth, we get (11) by letting $k \rightarrow \infty$. Finally, by its definition, $g \in L^{2}\left(\left(2 \tau_{0}-t\right) d t\right)$; in particular, we get (i) of Theorem 1.

This proves half of Theorem 1. Assume now that $S$ satisfies (i) and (ii) of Theorem 1.

Claim 2.4. For any $\tau \leq \tau_{0}$,

$$
\begin{aligned}
\sup _{n} \sum_{i}\left(\lambda_{i}^{n}(\tau)-1\right)^{2} \leq\left(\frac{S_{0}}{1-\tau}-1\right)^{2}+\int_{0}^{2 \tau} & S^{\prime \prime}(t)^{2}(2 \tau-t) d t \\
& +\frac{2}{1-\tau} \int_{0}^{2 \tau} S^{\prime}(t)^{2} d t<\infty .
\end{aligned}
$$

Proof. Note that, with $T=T_{n}(\tau)=\left(t_{i j}\right)_{0 \leq i, j \leq n}$,

$$
\sum_{i=1}^{n} t_{i 0}^{2}+t_{0 i}^{2}=\frac{2}{1-\tau} \sum_{i=1}^{n}\left(S_{i}-S_{i-1}\right)^{2} \frac{1}{\delta} \leq \frac{2}{1-\tau} \int_{0}^{2 \tau} S^{\prime}(t)^{2} d t
$$

Let

$$
\bar{S}(r) \stackrel{d}{=} S(r)-\left(1-\frac{|r|}{2}\right)
$$


Then $\bar{S}$ is an even function, with absolutely continuous derivative $\bar{S}^{\prime}$ on $\left(-2 \tau_{0}, 2 \tau_{0}\right)$, and $\bar{S}^{\prime \prime} \equiv S^{\prime \prime}$ on $\left(0,2 \tau_{0}\right)$. It is not hard to verify that

$$
\begin{aligned}
t_{i j} & =-\frac{1}{\delta} \int_{(i-1) \delta}^{i \delta} \int_{(j-1) \delta}^{j \delta} \bar{S}^{\prime \prime}(t-s) d t d s & 1 \leq i \neq j \leq n, \\
t_{i i}-1 & =-\frac{1}{\delta} \int_{(i-1) \delta}^{i \delta} \int_{(i-1) \delta}^{i \delta} \bar{S}^{\prime \prime}(t-s) d t d s & 1 \leq i \leq n .
\end{aligned}
$$

Thus, for $\tau \leq \tau_{0}$

$$
\begin{aligned}
{\left[\sum_{1 \leq i \neq j \leq n} t_{i j}^{2}+\sum_{i=1}^{n}\left(t_{i i}-1\right)^{2}\right] } & \leq \int_{0}^{2 \tau} \int_{0}^{2 \tau} \bar{S}^{\prime \prime}(t-s)^{2} d t d s \\
& =\int_{0}^{2 \tau} S^{\prime \prime}(t)^{2}(2 \tau-t) d t<\infty
\end{aligned}
$$

which together with (12) and (4) completes the proof of Claim 2.4.

We next show that for $\tau<\tau_{0}, \inf _{i, n} \lambda_{i}^{n}(\tau)>0$. It follows that,

$$
H^{\tau}=\varlimsup \sum \frac{\left(\lambda_{i}^{n}-1\right)^{2}}{\lambda_{i}^{n}} \leq \frac{1}{\inf _{i, n} \lambda_{i}^{n}(\tau)} \varlimsup \sum\left(\lambda_{i}^{n}-1\right)^{2}<\infty,
$$

which completes the proof of Theorem 1 .

Since $\bar{S}^{\prime \prime} \in L^{2}([0,2 \tau] \times[0,2 \tau])$, it defines a compact integral operator on $L^{2}(0,2 \tau)$ :

$$
\left(\bar{S}^{\prime \prime} * f\right)(r) \stackrel{d}{=} \int_{0}^{2 \tau} \bar{S}^{\prime \prime}(r-t) f(t) d t
$$

Let $\mathcal{H}=\mathcal{H}_{\tau} \stackrel{d}{=} \mathbb{R} \oplus L^{2}(0,2 \tau)$. Define a bounded symmetric operator $\mathcal{T}_{\tau}$ : $(\beta, f) \in \mathcal{H} \mapsto \mathcal{H}$ as follows:

$$
\mathcal{T}(\beta, f) \stackrel{d}{=}\left(\beta \frac{S(0)}{1-\tau}+\frac{1}{\sqrt{1-\tau}} \int_{0}^{2 \tau} S^{\prime}(t) f(t) d t, \frac{\beta}{\sqrt{1-\tau}} S^{\prime}+f-\bar{S}^{\prime \prime} * f\right) .
$$

Let $U_{n}$ be the $n$ dimensional subspace of $L^{2}(0,2 \tau)$ populated by functions which are constant on the intervals $[i \delta,(i+1) \delta), \delta=2 \tau / n$. For $f \in U_{n}$ and $X \in C[0,2 \tau]$, we define $\int_{0}^{2 \tau} f(t) d X_{t}$ in the obvious manner. Let ${ }^{\wedge}: U_{n} \mapsto \mathbb{R}^{n}$ be the natural isometry between these spaces, i.e., for

$$
\begin{gathered}
f(t)=\sum_{i=1}^{n} f_{i} 1_{[(i-1) \delta, i \delta)}(t) \in U_{n}, \\
\hat{f} \stackrel{d}{=}\left(f_{1} \sqrt{\delta}, f_{2} \sqrt{\delta}, \ldots, f_{n} \sqrt{\delta}\right) .
\end{gathered}
$$


Let $Q$ be a stationary extension of $Q^{\tau_{0}}$ to $C(\mathbb{R})$ corresponding to a positivedefinite extension of $S$ from $\left[-2 \tau_{0}, 2 \tau_{0}\right]$ to $\mathbb{R}$. A straight forward computation shows that for $f \in U_{n}$ and $\beta \in \mathbb{R}$,

$$
\langle T(\beta, \hat{f}),(\beta, \hat{f})\rangle_{\mathbb{R}^{n+1}}=E^{Q}\left[\frac{\beta}{\sqrt{1-\tau}} X_{0}+\int_{0}^{2 \tau} f(t) d X_{t}\right]^{2} .
$$

Remark. The last equation might deserve a second look: On the left hand side we have $T$ which was obtained from $S$ and $R$, while on the right hand side $R$ does not appear explicitly.

It follows from (13) that,

$$
\langle T(\beta, \hat{f}),(\beta, \hat{f})\rangle_{\mathbb{R}^{n+1}}=\langle\mathcal{T}(\beta, f),(\beta, f)\rangle_{\mathcal{H}} .
$$

Thus, for $\beta=0$ and $f \in U_{n}$,

$$
E^{Q}\left[\int_{0}^{2 \tau} f(t) d X_{t}\right]^{2}=\langle\mathcal{T}(0, f),(0, f)\rangle_{\mathcal{H}} \leq\|\mathcal{T}\||f|_{L^{2}}^{2}
$$

Hence the map

$$
f \mapsto \int_{0}^{2 \tau} f(t) d X_{t}
$$

defined initially on $\bigcup U_{n}$, can be extended uniquely as a bounded linear map from $L^{2}(0,2 \tau)$ into $L^{2}(\Omega, d Q)$, where $\Omega=C_{0}$ is our probability space. Furthermore, by continuity, for any $f \in L^{2}(0,2 \tau)$ and $\beta \in \mathbb{R}$,

$$
\langle\mathcal{T}(\beta, f),(\beta, f)\rangle_{\mathcal{H}}=E^{Q}\left[\frac{\beta}{\sqrt{1-\tau}} X_{0}+\int_{0}^{2 \tau} f(t) d X_{t}\right]^{2} .
$$

In particular, $\mathcal{T}$ is positive-definite.

Claim 2.5. Suppose that $\inf _{i, n} \lambda_{i}^{n}(\tau)=0$, then there exists $\beta \in \mathbb{R}$ and $f \in L^{2}(0,2 \tau)$, not identically 0 , with

$$
\frac{\beta}{\sqrt{1-\tau}} X_{0}+\int_{0}^{2 \tau} f(t) d X_{t}=0 \quad \text { a.s. } d Q .
$$

Proof. Assuming the eigenvalues of $T_{n}$ satisfy $\lambda_{0}^{n}(\tau) \leq \lambda_{1}^{n} \leq \ldots \lambda_{n}^{n}$, there exists a sub-sequence $n_{k}$ such that

$$
\nu_{k} \stackrel{d}{=} \lambda_{0}^{n_{k}} \longrightarrow 0 .
$$

Let $\boldsymbol{v}_{k}=\left(\boldsymbol{v}_{k}(0), \boldsymbol{v}_{k}(1), \ldots, \boldsymbol{v}_{k}\left(n_{k}\right)\right) \in \mathbb{R}^{n_{k}+1}$ be the corresponding normalized eigenvectors, i.e.,

$$
T_{n_{k}} \boldsymbol{v}_{k}=\nu_{k} \boldsymbol{v}_{k}, \quad\left|\boldsymbol{v}_{k}\right|=1 .
$$

Let $\beta_{k}=\boldsymbol{v}_{k}(0)$ and let

$$
f_{k} \stackrel{d}{=}\left(\boldsymbol{v}_{k}(1) \widehat{\ldots, \boldsymbol{v}_{k}}\left(n_{k}\right)\right) .
$$


Then $\left\|\left(\beta_{k}, f_{k}\right)\right\|_{\mathcal{H}}=1$, and without loss of generality you may assume that

$$
\left(\beta_{k}, f_{k}\right) \longrightarrow(\beta, f) \quad \text { weakly in } \mathcal{H}
$$

Let $\mathcal{I}$ be the identity in $\mathcal{H}$. The operator $\mathcal{T}_{0} \stackrel{d}{=} \mathcal{T}-\mathcal{I}$ is compact, by inspection, so

$$
\mathcal{T}_{0}\left(\beta_{k}, f_{k}\right) \longrightarrow \mathcal{T}_{0}(\beta, f) \quad \text { strongly in } \mathcal{H}
$$

It follows that

or

$$
\nu_{k}-1=\left\langle\mathcal{T}_{0}\left(\beta_{k}, f_{k}\right),\left(\beta_{k}, f_{k}\right)\right\rangle_{\mathcal{H}} \longrightarrow\left\langle\mathcal{T}_{0}(\beta, f),(\beta, f)\right\rangle_{\mathcal{H}},
$$

$$
\left\langle\mathcal{T}_{0}(\beta, f),(\beta, f)\right\rangle_{\mathcal{H}}=-1
$$

But $\mathcal{T}$ is symmetric and positive-definite, so the spectrum $\sigma\left(\mathcal{T}_{0}\right) \subset[-1, \infty)$, whence $\|(\beta, f)\|_{\mathcal{H}}=1$ and

$$
\langle\mathcal{T}(\beta, f),(\beta, f)\rangle_{\mathcal{H}}=0 .
$$

The claim now follows from (14).

\section{Remarks.}

- In hind sight $\left(\beta_{k}, f_{k}\right)$ converge strongly to $(\beta, f)$.

- $S$ has a unique extension as a symmetric positive-definite function from $[-2 \tau, 2 \tau]$ to $\mathbb{R}$. Indeed, let $\Delta$ be any spectral distribution function corresponding to such an extension of $S$. Using the standard isometry between $L^{2}(\mathbb{R}, \Delta)$ and $L^{2}(\Omega, Q)$, defined by

$$
e^{i t \omega} \longleftrightarrow X_{t},
$$

it follows from (15) that

$$
\frac{\beta}{\sqrt{1-\tau}}+i \omega \int_{0}^{2 \tau} f(t) e^{i \omega t} d t=0
$$

where the equality is in $L^{2}(\Delta)$. Thus, there exists a non-trivial holomorphic function of exponential type $\leq \tau$, which vanishes in $L^{2}(\Delta)$. Krein's alternative says that in this case the stationary process governed by any extension of $S$ must be completely predictable from any interval of length $2 \tau$ [2] [see Sec. 4.8]. This implies that there exists only one extension ([4] is helpful).

Claim 2.6. If (15) holds with a non-trivial $f \in L^{2}(0,2 \tau)$, then there exists $\tau^{\prime} \in\left(\tau, \tau_{0}\right)$, such that the eigenspace,

$$
V \stackrel{d}{=}\left\{g \in L^{2}\left(0,2 \tau^{\prime}\right): \int_{0}^{2 \tau^{\prime}} \bar{S}^{\prime \prime}(t-r) g(r) d r=g(t)\right\},
$$

is infinite-dimensional. 
Proof. Since $Q$ is stationary, it follows from (15) that for any $\delta>0$,

$$
\frac{\beta}{\sqrt{1-\tau}} X_{2 \delta}+\int_{0}^{2 \tau} f(t) d X_{t+2 \delta}=0 \quad \text { a.s. } d Q .
$$

Let

$$
g(t) \stackrel{d}{=} \begin{cases}\frac{\beta}{\sqrt{1-\tau}}-f(t) & 0 \leq t<2 \delta \\ f(t-2 \delta)-f(t) & 2 \delta \leq t<2 \tau \\ f(t-2 \delta) & 2 \tau \leq t<2 \tau+2 \delta \\ 0 & \text { elsewhere }\end{cases}
$$

Subtracting (15) from (16), we find

$$
\int_{0}^{2(\tau+\delta)} g(t) d X_{t}=0 \quad \text { a.s. } d Q .
$$

Fix $\tau^{\prime} \in\left(\tau, \tau_{0}\right)$ and choose $\delta>0$ so small that $\tau+\delta<\tau^{\prime}$. By (14),

$$
\left\langle\mathcal{T}_{\tau^{\prime}}(0, g),(0, g)\right\rangle_{\mathcal{H}_{\tau^{\prime}}}=E^{Q}\left[\int_{0}^{2 \tau^{\prime}} g(t) d X_{t}\right]^{2}=0 .
$$

For any $\alpha>0$ such that $\tau+\delta+\alpha<\tau^{\prime}$, let $g_{\alpha}(t) \stackrel{d}{=} g(t-\alpha)$. By stationarity,

$$
\left\langle\mathcal{T}_{\tau^{\prime}}\left(0, g_{\alpha}\right),\left(0, g_{\alpha}\right)\right\rangle_{\mathcal{H}_{\tau^{\prime}}}=E^{Q}\left[\int_{0}^{2 \tau^{\prime}} g(t-\alpha) d X_{t}\right]^{2}=0
$$

But $\mathcal{T}_{\tau^{\prime}}$ is symmetric and positive-definite, so

$$
\mathcal{T}_{\tau^{\prime}}\left(0, g_{\alpha}\right)=0
$$

which implies

$$
g_{\alpha}=\bar{S}^{\prime \prime} * g_{\alpha}
$$

These $\alpha$-translates of $g$ span an infinite dimensional subspace $\subset V$.

The next lemma shows that $V$ cannot be continuously embedded in $L^{\infty}\left(0,2 \tau^{\prime}\right)$.

Lemma 2.7. Let $U$ be an infinite-dimensional subspace of $L^{2}(0, T)$, with $T<\infty$. Then $U$ cannot be continuously embedded in $L^{\infty}(0, T)$. 


\section{Remarks.}

- Undoubtedly, this lemma is known. However having no citation to provide, we give here a proof.

- $T<\infty$ is crucial here. Assume without loss of generality that $T=1$.

Proof. Let $\left\{\varphi_{n}\right\}$ be an orthonormal set in $U$. If $M \stackrel{d}{=} \sup _{n}\left\|\varphi_{n}\right\|_{\infty}$ is infinite, we are done, so assume $M<\infty$. Let

$$
\begin{aligned}
& A_{n} \stackrel{d}{=}\left\{x \in[0, T]: \varphi_{n}(x)>\frac{1}{\sqrt{2}}\right\}, \\
& B_{n} \stackrel{d}{=}\left\{x \in[0, T]: \varphi_{n}(x)<-\frac{1}{\sqrt{2}}\right\} .
\end{aligned}
$$

Let $\lambda(A)$ be the Lebesgue measure of $A$. Then

$$
\lambda\left(A_{n} \cup B_{n}\right) \geq \frac{1}{2 M^{2}}=2 \alpha>0 .
$$

We can assume, without loss of generality, that $\lambda\left(A_{n}\right) \geq \alpha$ for all $n$. Let $N \stackrel{d}{=}[n / \alpha+1]$ and let

$$
\varphi(x) \stackrel{d}{=} \sum_{k=1}^{N} 1_{A_{k}}(x)
$$

Then

$$
\int_{0}^{1} \varphi(x) d x \geq N \alpha
$$

Necessarily,

$$
\lambda\left(\left\{x: \sum_{k=1}^{N} 1_{A_{k}} \geq n\right\}\right)>0
$$

whence there are $n$ indices, $k_{1}, \ldots, k_{n}$, such that

$$
\lambda\left(A_{k_{1}} \cap A_{k_{2}} \cap \cdots \cap A_{k_{n}}\right)>0 .
$$

Now

$$
\left\|\sum_{j=1}^{n} \frac{1}{\sqrt{n}} \varphi_{k_{j}}\right\|_{\infty}>\sqrt{\frac{n}{2}} \quad \text { but } \quad\left\|\sum_{j=1}^{n} \frac{1}{\sqrt{n}} \varphi_{k_{j}}\right\|_{2}=1,
$$

which completes the proof.

The proof that $\inf _{i, n} \lambda_{i}^{n}(\tau)>0$ is now complete: else, by Claims 2.5 and 2.6 there exists $\tau^{\prime} \in\left(\tau, \tau_{0}\right)$ and an infinite dimensional eigenspace $V$ as described in Claim 2.6. Hence by the last lemma, there exist a sequence $f_{n} \in V$ such that,

$$
\left\|f_{n}\right\|_{L^{2}}=1, \quad f_{n}=\bar{S}^{\prime \prime} * f_{n} \quad \text { and } \quad\left\|f_{n}\right\|_{\infty}>n .
$$


But this implies $\bar{S}^{\prime \prime} \notin L^{2}\left(0,2 \tau^{\prime}\right)$, contradicting the assumption that (i) holds.

\section{References}

[1] N.I. Akhiezer, The Classical Moment Problem and Some Related Questions in Analysis, Hafner Publishing Company, 1965.

[2] H. Dym and H.P. McKean, Gaussian Processes, Function Theory, and the Inverse Spectral Problem, Academic Press, 1976.

[3] T. Hida and M. Hitsuda, Gaussian Processes, AMS, 1993.

[4] M.G. Krein, On the continuation problem for Hermitian-positive continuous functions, Dokl. Akad. Nauk SSSR, 26 (1940), 17-21.

[5] L.A. Shepp, Radon-Nikodym derivatives of Gaussian measures, Ann. Math. Stat., 37 (1966), 321-354.

Received July 6, 1998.

CALTech

Pasadena, CA 91125

E-mail address: keich@ama.caltech.edu 